\title{
Sijunzi decoction may decrease apoptosis via stabilization of the extracellular matrix following cerebral ischaemia-reperfusion in rats
}

\author{
PING YANG ${ }^{1}$, YE-MEI TIAN ${ }^{2}$, WEN-XIANG DENG ${ }^{2}$, XIONG CAI $^{2}$, \\ WANG-HUA LIU ${ }^{2}$, LIANG $\mathrm{LI}^{2,3^{*}}$ and HUI-YONG HUANG ${ }^{2 *}$ \\ ${ }^{1}$ Department of Psychiatry, Brains Hospital of Hunan Province, \\ Clinical Medical School, Hunan University of Chinese Medicine, Changsha, Hunan 410007; \\ ${ }^{2}$ Provincial Key Laboratory of TCM Diagnostics; ${ }^{3}$ Key Discipline of Anatomy and Histoembryology, \\ Hunan University of Chinese Medicine, Changsha, Hunan 410208, P.R. China
}

Received February 3, 2018; Accepted June 13, 2019

DOI: $10.3892 / e t m .2019 .7878$

\begin{abstract}
Neurons undergo degeneration, apoptosis and death due to ischaemic stroke. The present study investigated the effect of Sijunzi decoction (SJZD), a type of traditional Chinese medicine known as invigorating spleen therapy, on anoikis (a type of apoptosis) in rat brains following cerebral ischaemia-reperfusion. Rats were randomly divided into sham, model, nimodipine and SJZD low/medium/high dose groups. A middle cerebral artery occlusion model was established. Neurobehavioural scores were evaluated after administration for 14 days using a five-grade scale. Blood-brain barrier permeability and apoptotic rate were detected using Evans blue (EB) extravasation and TUNEL staining, respectively. Tissue inhibitor of metalloproteinase 1 (TIMP-1), matrix metalloproteinase 9 (MMP-9) and collagen IV (COL IV) were determined using immunohistochemistry. Neurobehavioural scores decreased remarkably in all SJZD and nimodipine groups compared to the model group $(\mathrm{P}<0.05)$. Compared with the sham group,
\end{abstract}

Correspondence to: Professor Hui-Yong Huang or Dr Liang Li, Provincial Key Laboratory of TCM Diagnostics, Hunan University of Chinese Medicine, 300 Xueshi Road, Science and Education Park, Yuelu, Changsha, Hunan 410208, P.R. China

E-mail: superliliang@126.com

E-mail: huanghy68@126.com

${ }^{*}$ Contributed equally

Abbreviations: ECM, extracellular matrix; SJZD, Sijunzi decoction; TIMP-1, tissue inhibitor of metalloproteinase 1; MMP-9, matrix metalloproteinase 9; COL IV, collagen IV; MCAO, middle cerebral artery occlusion; EB, Evans blue; BBB, blood-brain barrier; TCM, Traditional Chinese Medicine; CNS, central nervous system

Key words: apoptosis, cerebral ischaemia-reperfusion, extracellular matrix, Sijunzi decoction, tissue inhibitor of metalloproteinase 1, matrix metalloproteinase 9, collagen IV
EB extravasation was higher in the model group $(\mathrm{P}<0.01)$. The amount of EB extravasation decreased in the SJZD high dose and nimodipine groups compared to the model group $(\mathrm{P}<0.01)$, and extravasation in the SJZD high dose group was lower than the SJZD low and medium dose groups $(\mathrm{P}<0.01)$. TIMP-1 and MMP-9 expression and apoptotic rate increased, but COL IV decreased significantly in the hippocampus of the model group compared to the sham group $(\mathrm{P}<0.01)$. TIMP-1 and COL IV expression increased significantly and MMP-9 and apoptotic rate decreased remarkably in all SJZD and nimodipine groups compared to the model group $(\mathrm{P}<0.01)$. TIMP-1 and COL IV expression decreased, but MMP-9 expression and apoptotic rate increased in the SJZD low and medium dose groups compared to the SJZD high dose group $(\mathrm{P}<0.01)$. SJZD rescued neurons and improved neurobehavioural function in rats following cerebral ischaemia-reperfusion, especially when used at a high dose. The mechanism may be related to protection of the extracellular matrix followed by anti-apoptotic effects.

\section{Introduction}

Ischaemic stroke is a common disease in the central nervous system (CNS), and it occurs with high incidence, mortality and disability rates (1-3). Neuronal apoptosis following cerebral ischaemia-reperfusion leads to brain injury and neurological impairment (4). In recent years, studies have found that anoikis is a substantial path for neuron apoptosis $(5,6)$. Anoikis is programmed cell death that occurs due to cell detachment from the extracellular matrix (ECM) (7). The ECM provides an external environment and support for cell survival $(8,9)$. Enzymes degrade the ECM during the cerebral ischaemia-reperfusion process, leading to maladjustments in signal transmission between cells, neuronal injury and apoptosis $(10,11)$.

In Traditional Chinese Medicine (TCM), it is believed that the spleen is the foundation of life and the source of Qi and blood generation. Sijunzi decoction (SJZD) is one of the most famous invigorating spleen therapy recipes in TCM. This Chinese decoction has been used to treat gastrointestinal 
disorders, including chronic gastritis and gastroduodenal ulcer, for hundreds of years, and it is effective for nausea, vomiting and diarrhoea $(12,13)$. SJZD was not created for the treatment of stroke initially, but an increasing number of studies have found that SJZD is beneficial for stroke, For example, a study found that SJZD could protect cerebral neurons via up-regulating p-ERK1/2 and p-Akt, and inhibiting the expression of Bax protein (14). Another study demonstrated that it significantly increased the expression of superoxide dismutase and decreased malondialdehyde in rat brains, and thus protected the cerebral ischemia-damaged neurons (15). One of the most commonly used models for studying stroke in rats is the middle cerebral artery occlusion (MCAO) model (16). $\mathrm{MCAO}$ is one of the most severe types of stroke in clinic and the most common type of ischemia (17). During the process of model establishment in the current study, the MCA was occluded with a filament, resulting in focal infarct to the ipsilateral hemisphere.

The present study examined the anti-apoptotic effect of SJZD in rats, following cerebral ischaemia-reperfusion. Nimodipine is an effective medicine for the treatment of stroke, and therefore was used as positive control treatment in the current study. Tissue inhibitor of metalloproteinase 1 (TIMP-1), matrix metalloproteinase 9 (MMP-9), collagen IV (COL IV), cell apoptotic rate and the blood-brain barrier (BBB) were examined. The results of the present study may highlight a neuroprotective effect of SJZD in cerebral ischaemia-reperfusion.

\section{Materials and methods}

Animals and experimental design. The present study was a randomized, controlled animal experiment, performed at the Hunan Provincial Key Laboratory of Diagnostics in Chinese Medicine (Changsha, China). A total of 150 male Sprague-Dawley rats (weight, 250-280 g; age, 10-11 weeks) were supplied by the Laboratory Animal Centre of Hunan University of Chinese Medicine [license no. SYXK (Xiang) 2013-0005; Changsha, China]. The rats were housed separately in plastic cages at $22^{\circ} \mathrm{C}$ and $60 \%$ humidity under a light-dark cycle (12 $\mathrm{h}$ for each) and allowed free access to food and water. The animals were randomly divided into the following six groups ( $n=25$ per group): Sham group and untreated MCAO model group, nimodipine MCAO model group $(10.8 \mathrm{mg} / \mathrm{kg})$, and SJZD low dose $(3 \mathrm{~g} / \mathrm{kg})$, SJZD medium dose $(6 \mathrm{~g} / \mathrm{kg})$ and SJZD high dose $(9 \mathrm{~g} / \mathrm{kg})$ MCAO model groups. The nimodipine and SJZD groups received corresponding treatment administrated via gastric gavage once daily for 14 days after model establishment. The animals in the sham and untreated model groups were administered $0.9 \%$ saline solution only via gastric gavage once daily for 14 days after model establishment. The present study was approved by the Brains Hospital of Hunan Province Ethics Committee (permit number, Z2015003; Changsha, China). All procedures were performed in accordance with the Guidance Suggestions for the Care and Use of Laboratory Animals, formulated by the Ministry of Science and Technology of China.

Chemicals and reagents. SJZD is composed of Radix et Rhizoma Ginseng, Rhizome Atractylodis macrocephala,
Wolfiporia extensa and Radix et Rhizoma Glycyrrhizae (the ratio was 3:3:3:2). All herbs (from Good Agricultural Practice planting base) were purchased from LBX Pharmacy Chain Co., Ltd. All ingredients were identified by experts from the School of Pharmacy, Hunan University of Chinese Medicine to fulfil the quality requirements of the Pharmacopoeia of the People's Republic of China (18). The mixture was decocted in boiling water for $45 \mathrm{~min}$ and vacuum-dried to yield a final concentration of $2 \mathrm{~g}$ crude $\mathrm{drug} / \mathrm{ml}$. The crude drug was dissolved in distilled water and administered at 3,6 and $9 \mathrm{~g} / \mathrm{kg}$ in accordance with a preliminary pharmacology experiment performed in Provincial Key Laboratory of TCM Diagnostics (Hunan University of Chinese Medicine, Changsha, China).

Nimodipine tablets (20 mg; cat. no. BG28956; Bayer AG) were dissolved in distilled water and $10.8 \mathrm{mg} / \mathrm{kg}$ was administered in accordance with pharmacology experiments performed in Provincial Key Laboratory of TCM Diagnostics.

Polyclonal rabbit antibodies against TIMP-1 (cat. no. 10753-1-AP), MMP-9 (cat. no., 10375-2-AP) and COL IV (cat.no. 24460-1-AP) were purchased from ProteinTech Group, Inc. The PV-9000 2-step plus poly-HRP anti-mouse/rabbit IgG Detection system (cat. no. WP140316) and DAB kit (cat. no. K145619C) were purchased from Beijing Zhongshan Golden Bridge Co., Ltd. Evans blue (EB; cat. no. 46160) was purchased from Sigma-Aldrich; Merck KGaA. The TUNEL detection kit for assessing cell apoptosis (cat. no. KGA7023-A) was purchased from Nanjing KeyGen Biotech Co., Ltd.

MCAO model establishment. The MCAO model was established as described previously (19). Rats were anaesthetized by intraperitoneal (i.p.) injection of $2 \%$ pentobarbital sodium (30 $\mathrm{mg} / \mathrm{kg}$ ), and placed in a supine position. The left common carotid artery (CCA), external carotid artery (ECA) and internal carotid artery (ICA) were exposed and isolated carefully. The CCA and ICA were clipped using artery clamps. The ECA was ligated and cut $\sim 5 \mathrm{~mm}$ from the CCA bifurcation. A 0.26-mm-diameter nylon filament (tip diameter $0.36 \pm 0.02 \mathrm{~mm}$; Beijing Sunbio Biotech Co., Ltd.) was inserted through the ECA stump and gently advanced $10 \mathrm{~mm}$ to occlude the origin of the middle cerebral artery. The filament was removed to restore blood flow after $1.5 \mathrm{~h}$ of MCAO (reperfusion). Rats in the sham group underwent the same surgical procedure, however the filament was not inserted as far as the CCA bifurcation. The rats were housed individually and allowed to recover. When they woke up, Horner's syndrome was observed. One of the characteristics of Horner's syndrome is partial ptosis that occurs on the same side (ipsilateral) (20). Neurobehavioural scores were assessed using a five-grade scale (21) $2 \mathrm{~h}$ after model establishment and $14 \mathrm{~d}$ after administration of treatment, and the severity of neurological symptoms was graded on a scale of 0-4 with slight modifications: 0 , no functional impairment; 1, cannot extend right forepaw; 2, whirls to the right; 3, leans to the right while walking; and 4, no autonomic activities in combination with being unconscious. Rats with scores $<2$ were excluded. The behavioural observations were performed in a blinded manner.

Evaluation of $B B B$ integrity. BBB integrity was assessed by determining $\mathrm{EB}$ extravasation in 8 rats per group. $\mathrm{EB}$ dye ( $2 \%$ in saline, $4 \mathrm{ml} / \mathrm{kg}$ ) was injected into the right femoral vein 
$30 \mathrm{~min}$ before reperfusion. Rats were deeply anaesthetized with $2 \%$ pentobarbital sodium $(30 \mathrm{mg} / \mathrm{kg}$; i.p.), perfused with PBS through the left ventricle to remove the intravascular dye until a colourless perfusion fluid was observed from the right atrium. Rats were executed by cervical dislocation. The hippocampus of the ischaemic side was removed quickly, weighed and homogenized in $2.5 \mathrm{ml}$ PBS. The homogenized solution was mixed with $2.5 \mathrm{ml}$ trichloroacetic acid (60\%) and centrifuged at $1,640 \mathrm{x} \mathrm{g}$ for $20 \mathrm{~min}$ at $4^{\circ} \mathrm{C}$. The supernatant was used to determine EB absorbance at $620 \mathrm{~nm}$ using a spectrophotometer. EB concentrations were calculated against a standard curve, and the obtained results were expressed as $\mu \mathrm{g} / \mathrm{g}$ brain tissue.

Brain slice preparation. Rats were anaesthetized as aforementioned after 14 days of treatment administration, perfused through the heart with $0.9 \%$ saline solution (100 $\mathrm{ml}$ ) followed by $4 \%$ paraformaldehyde in $0.1 \mathrm{M}$ phosphate buffered saline, $\mathrm{pH} 7.4$ at $4^{\circ} \mathrm{C}(150 \mathrm{ml})$. The brain was removed immediately and cut coronally at the levels of the optic chiasma and the posterior pole of the cerebrum. The area between these two coronal sections was kept for immunohistochemistry and TUNEL staining. A total of 8 samples from each group were used for paraffin sectioning and detection of COL IV expression. The section thickness was $5 \mu \mathrm{m}$. For TUNEL staining and detection of TIMP-1 and MMP-9 expression, 9 samples from each group were cut with a Leica VT1200S vibrating-blade microtome (Leica Microsystems GmbH). Tissue blocks were adhered vertically to the specimen holder of the vibrating-blade microtome. The clearance angle was adjusted to $18^{\circ}$, the blade holder sectioning speed was controlled with a motor at $0.5 \mathrm{~mm} / \mathrm{s}$, the amplitude was $2 \mathrm{~mm}$, and the thickness of each slice was $30 \mu \mathrm{m}$. Slices were collected in 24-well plates for TUNEL staining and for detecting the expression of TIMP-1 and MMP-9.

Immunohistochemistry. TIMP-1, MMP-9 and COL IV expression was measured using immunohistochemistry. Sections were immersed in hydrogen peroxide $(0.3 \%$ in PBS) for $10 \mathrm{~min}$ and incubated overnight at $4^{\circ} \mathrm{C}$ with the following polyclonal rabbit anti-rat antibodies: TIMP-1 (1:100), MMP-9 (1:100) and COL IV (1:150). Slices were incubated for $30 \mathrm{~min}$ at $37^{\circ} \mathrm{C}$ with ready-to-use poly-HRP anti-mouse/rabbit IgG and detected using the DAB kit for $15 \mathrm{~min}$ at $37^{\circ} \mathrm{C}$. Brownish yellow particles in the nucleus or cytoplasm of cells in the hippocampus indicated positive expression for TIMP-1 and MMP-9 proteins, and the presence of these particles in the ECM indicated positive staining for COL IV. A total of four sections from each specimen were imaged, and six visual fields were selected randomly in each section. Slices were observed under an optical microscope (Motic B5; Motic China Group Co., Ltd.) at a magnification of $x 400$. The average optical density (AOD) was calculated with Image-Pro software (version 6.0.0.260; Media Cybernetics Inc.).

TUNEL staining. TUNEL staining was performed to quantify cell apoptosis using a TUNEL Detection kit, according to the manufacturer's instructions. Briefly, sections were fixed with $4 \%$ paraformaldehyde at $37^{\circ} \mathrm{C}$ for $20 \mathrm{~min}$, immersed in the $3 \%$
$\mathrm{H}_{2} \mathrm{O}_{2}$ at $37^{\circ} \mathrm{C}$ for $10 \mathrm{~min}$, and dipped into $0.1 \%$ TritonX-100 on ice for $2 \mathrm{~min}$. Biotin-dUTP $(50 \mu \mathrm{l})$ from the kit was added to each section and then incubated at $37^{\circ} \mathrm{C}$ for $60 \mathrm{~min}$, followed by incubation with $50 \mu 1$ streptavidin-HRP from the kit at $37^{\circ} \mathrm{C}$ for $30 \mathrm{~min}$. After detection with the DAB kit for $10 \mathrm{~min}$ at $37^{\circ} \mathrm{C}$, sections were sealed with neutral gum. Six images of randomly selected visual fields were taken for each section with a Motic B5 optical microscope at x400 magnification. TUNEL-positive cells in the ipsilateral hippocampus were observed and quantified with Image-Pro software (version 6.0.0.260). Brownish yellow particles in the nuclei of the cells in the hippocampal sections indicated TUNEL-positive, apoptotic cells. The apoptotic rate was calculated quantified with Image-Pro software (version 6.0.0.260).

Statistical analysis. Statistical analysis was performed using SPSS software (version 24.0; IBM Corp.). Data were analysed using one-way ANOVA followed by the least significant difference test or Dunnett's T3 test. The results are expressed as the mean \pm SEM. $\mathrm{P}<0.05$ was considered to indicate a statistically significant difference.

\section{Results}

Status of rats following MCAO model establishment. In the untreated MCAO model group, 2 rats died on days 2 and 5 after surgery due to subarachnoid haemorrhage and cerebral oedema induced by ischaemia, respectively. In the nimodipine group, 1 rat died on day 6 after surgery due to subarachnoid haemorrhage. In the SJZD low dose group, 2 rats died on days 3 and 4 after surgery due to subarachnoid haemorrhage and cerebral oedema, respectively. In the SJZD medium dose group, 2 rats died of subarachnoid haemorrhage on days 2 and 4 after surgery. No neurobehavioural scores of the surviving rats were $<2$, and no rats were excluded.

SJZD treatment improves neurobehavioural scores following MCAO. No neurobehavioural abnormalities of the contralateral limb were observed in the sham group. Horner's syndrome could be observed in rats in the model groups. These rats exhibited paralysis of the contralateral limb, turned or rotated to the contralateral side, limped, and appeared lethargic and inactive. Rats failed to abduct their contralateral forelimb when their tails were held or were not able to independently move their contralateral limbs and demonstrated spontaneous rotation to the contralateral side. The sham group had neurobehavioural scores of 0 . The neurobehavioural scores were markedly higher in the model group compared with the sham group $(\mathrm{P}<0.01)$, and were significantly decreased in the SJZD- and nimodipine-treated groups compared with the untreated model group $(\mathrm{P}<0.05$; Fig. 1).

High-dose SJZD treatment decreases EB extravasation following MCAO. As shown in Fig. 2, EB extravasation was significantly increased in the model group compared with the sham group $(\mathrm{P}<0.01)$. Compared with the untreated model group, EB extravasation decreased in the SJZD high-dose and nimodipine groups (both $\mathrm{P}<0.01$ ). EB extravasation in the SJZD low and medium-dose groups was not significantly 


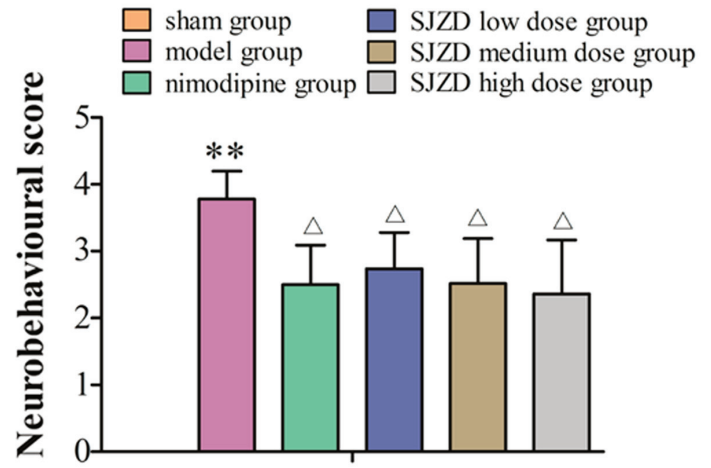

Figure 1. Neurobehavioural scores of rats following middle cerebral artery occlusion. ${ }^{* *} \mathrm{P}<0.01$ vs. sham group; ${ }^{\triangle} \mathrm{P}<0.05$ vs. model group. SJZD, Sijunzi decoction.

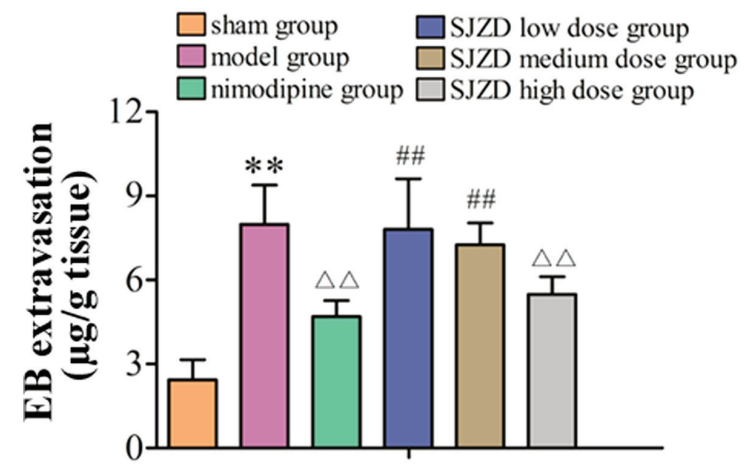

Figure 2. EB extravasation in the hippocampus of rats following middle cerebral artery occlusion. ${ }^{* *} \mathrm{P}<0.01$ vs. sham group; ${ }^{\Delta \Delta} \mathrm{P}<0.01$ vs. model group; ${ }^{\# \#} \mathrm{P}<0.01$ vs. SJZD high-dose group. SJZD, Sijunzi decoction; EB, Evans blue.

different to that in the model group. Extravasation in the SJZD high-dose group was lower than that in the SJZD low- and medium-dose groups $(\mathrm{P}<0.01)$.

SJZD treatment impacts expression of TIMP-1, MMP-9 and COL IV in the hippocampus following MCAO. Immunohistochemistry revealed TIMP-1- and MMP-9-positive cells in the hippocampus (Figs. 3 and 4). TIMP-1 and MMP-9 expression increased significantly in the hippocampus of the model group compared with the sham group $(\mathrm{P}<0.01$; Fig. 6$)$. COL IV decreased in the hippocampus of the model group compared with the sham group ( $\mathrm{P}<0.01$; Figs. 5 and 6). TIMP-1 and COL IV expression increased significantly, and MMP-9 expression decreased significantly in all SJZD and nimodipine groups compared with the untreated model group $(\mathrm{P}<0.01)$. TIMP-1 and COL IV expression decreased, and MMP-9 expression increased in the SJZD low- and medium-dose groups compared with the SJZD high-dose group $(\mathrm{P}<0.01$; Fig. 6).

SJZD treatment decreases the apoptotic rate in the hippocampus following MCAO. TUNEL staining identified apoptotic cells in the hippocampus (Fig. 7). The apoptotic rate increased in the hippocampus of the model group compared to the sham group, and SJZD and nimodipine treatment decreased apoptotic rate compared with that observed in the untreated model group. The apoptotic rate was significantly

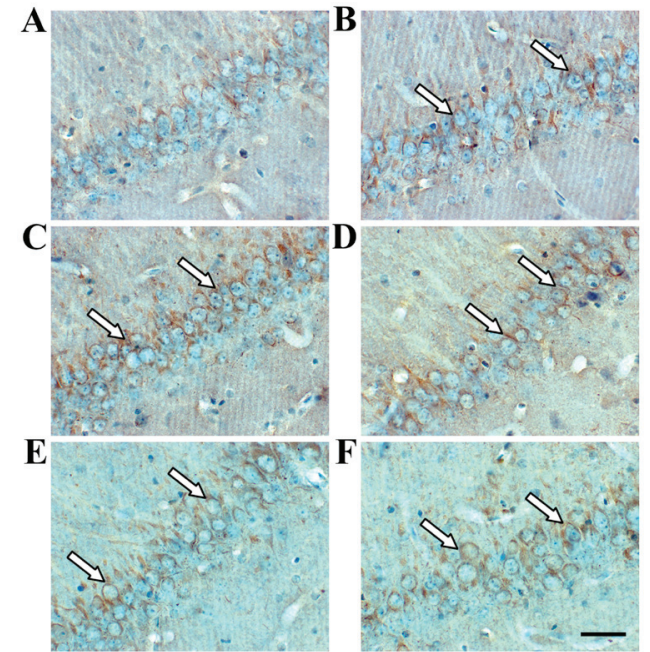

Figure 3. TIMP-1 expression in the hippocampus of rats following middle cerebral artery occlusion. Brownish yellow particles were observed in the nucleus or cytoplasm; arrows indicate positive TIMP-1 expression. Representative immunohistochemistry images of the (A) sham, (B) model, (C) nimodipine, (D) SJZD low-dose, (E) SJZD medium-dose and (F) SJZD high-dose groups. Scale bar, $50 \mu \mathrm{m}$; magnification, x400. SJZD, Sijunzi decoction; TIMP-1, tissue inhibitor of metalloproteinase 1.

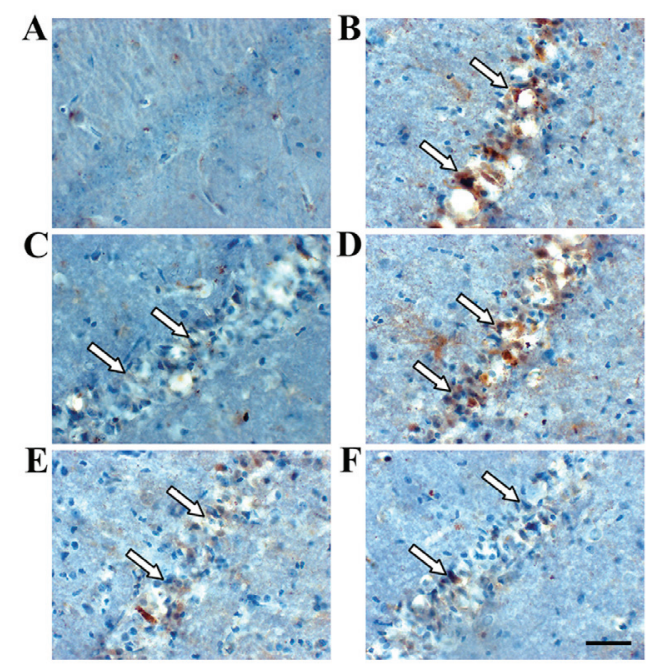

Figure 4. MMP-9 expression in the hippocampus of rats following middle cerebral artery occlusion. Brownish yellow particles were observed in the nucleus or cytoplasm; arrows indicate positive MMP-9 expression. Representative immunohistochemistry images of the (A) sham, (B) model, (C) nimodipine, (D) SJZD low-dose, (E) SJZD medium-dose and (F) SJZD high-dose groups. Scale bar, $50 \mu$ m; magnification, x400. SJZD, Sijunzi decoction; MMP-9, matrix metalloproteinase 9.

lower in the SJZD high-dose group than in the low- and medium-dose groups (Fig. 8).

\section{Discussion}

In TCM, most doctors choose classic methods to treat stroke, including blood-activating stasis removal, replenishment of Qi and blood, acupuncture, heat dissipation and detoxification (22-26). Our previous studies found that modified SJZD improved neurobehavioural function, decreased the neuronal apoptosis index and MMP-2 expression, and 


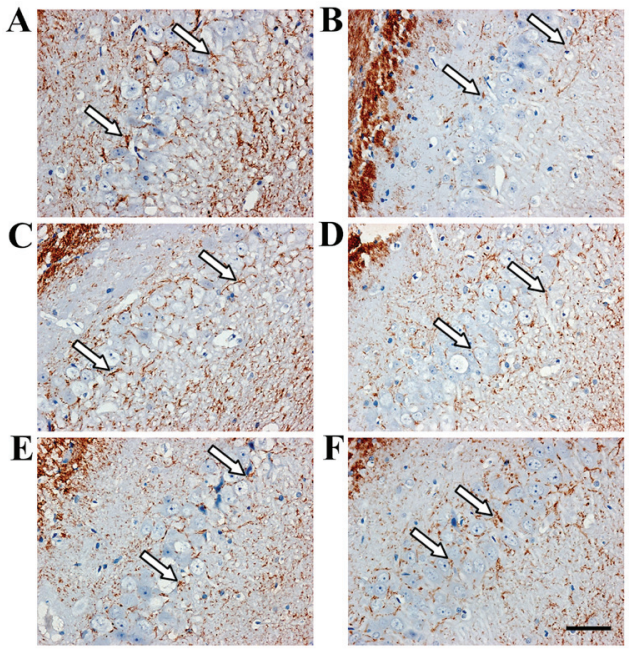

Figure 5. COL IV expression in the hippocampus of rats following middle cerebral artery occlusion. Brownish yellow particles were observed surrounding the neurons; arrows indicate positive expression of COL IV. Representative immunohistochemistry images of the (A) sham, (B) model, (C) nimodipine, (D) SJZD low-dose, (E) SJZD medium-dose and (F) SJZD high-dose groups. Scale bar=50 $\mu \mathrm{m}$; magnification, x400. SJZD, Sijunzi decoction; COL IV, collagen IV.

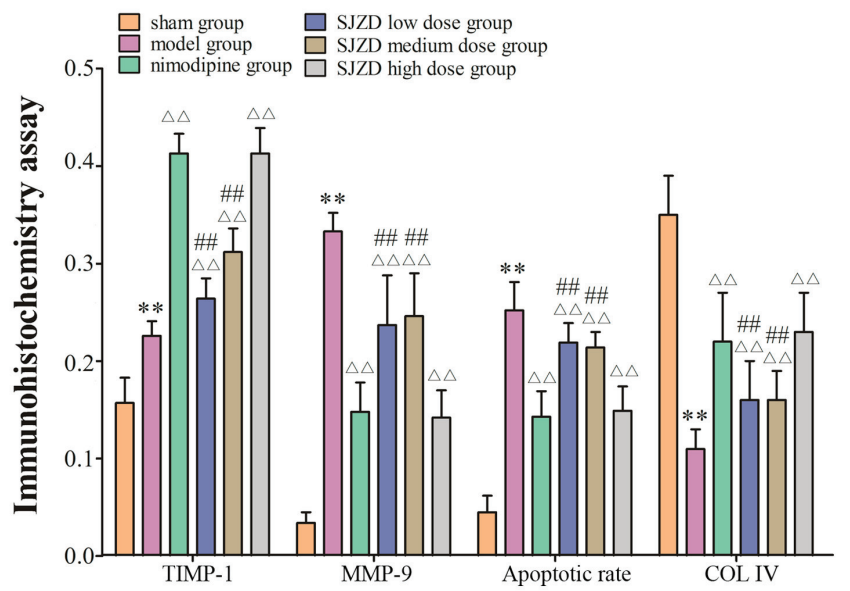

Figure 6. Expression of TIMP-1, MMP-9 and COL IV in the hippocampus of rats following middle cerebral artery occlusion. ${ }^{* *} \mathrm{P}<0.01$ vs. sham group; ${ }^{\triangle \Delta} \mathrm{P}<0.01$ vs. model group; ${ }^{\# \#} \mathrm{P}<0.01$ vs. SJZD high-dose group. SJZD, Sijunzi decoction; TIMP-1, tissue inhibitor of metalloproteinase 1; MMP-9, matrix metalloproteinase 9; COL IV, collagen IV.

increased laminin expression in the ECM after cerebral ischaemia-reperfusion (27-29). However, the mechanism underlying this therapeutic effect is not known. Damage and degradation of the ECM plays a key role during cerebral ischaemia-reperfusion (30-32).

The ECM is composed of a conglomerate assembly of proteins and proteoglycans, and it provides mechanical support and physical strength to the integrity of tissues, organs and the entire body $(33,34)$. The ECM is necessary for the CNS, as it unites neurons and glial cells to form its basic structure $(35,36)$. The triggering of intracellular and extracellular proteolytic processes during cerebral ischaemia often results in ECM degradation, which leads to oedema, haemorrhage, glial cell activation, inflammatory cell infiltration and neuronal injury (37). The ECM is also a constituent of the BBB $(38,39)$.

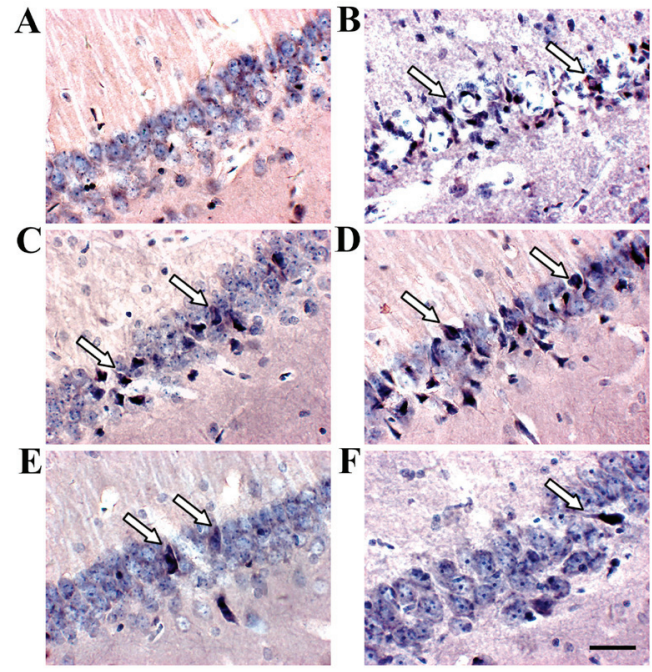

Figure 7. TUNEL staining expression in the hippocampus of rats following middle cerebral artery occlusion. Deep blue granules appeared in the nucleus, accompanied by concentrated nuclear chromatin or apoptotic bodies; arrows indicate apoptotic cells. Representative images of the (A) sham, (B) model, (C) nimodipine, (D) SJZD low-dose, (E) SJZD medium-dose and (F) SJZD high-dose groups. Scale bar, $50 \mu \mathrm{m}$; magnification x400. SJZD, Sijunzi decoction.

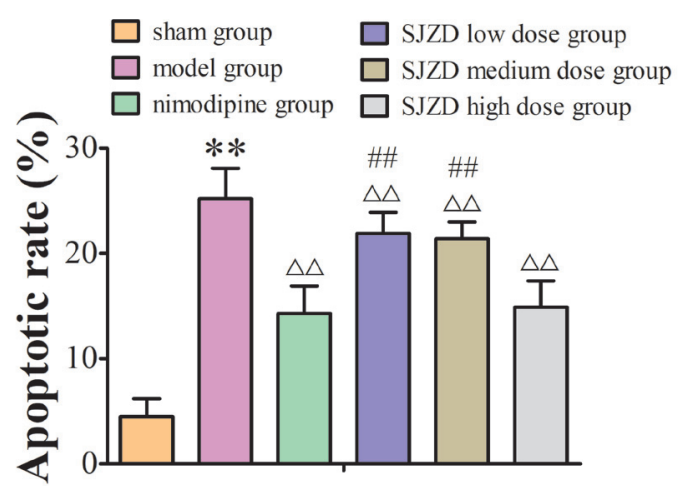

Figure 8. Apoptotic rate of the hippocampal neurons in rats following middle cerebral artery occlusion. ${ }^{* *} \mathrm{P}<0.01$ vs. sham group; ${ }^{\Delta \Delta} \mathrm{P}<0.01$ vs. model group; ${ }^{\# \#} \mathrm{P}<0.01$ vs. SJZD high-dose group. SJZD, Sijunzi decoction.

The BBB maintains the stability of the internal environment in the CNS (40), and it is composed of vascular endothelial cells, tight junctions of endothelial cells, basement membrane and the end feet of surrounding astrocytes $(41,42)$. COL IV is a major component of the basement membrane $(43,44)$. It offers adequate adhesion for nerve growth as it guides the nerve fibre to grow directionally along the matrix bridge (45). In the present study, it was hypothesised that SJZD treatment may protect against damage due to cerebral ischaemia by stabilising the ECM.

The primary components of the basement membrane are substrates of MMP-9, which is involved in the regulation of nearly all ECM components $(46,47)$. A previous study demonstrated that MMPs usually exist as proenzymes in the CNS (48). Activated MMPs destroy the tight junctions of endothelial cells, the basement membrane of capillaries and cerebrovascular integrity, which results in aseptic inflammation and BBB destruction (49). These changes cause blood 
constituent extravasation, vasogenic brain oedema and cerebral haemorrhage (50). MMP-9 activates other MMPs, which leads to a waterfall effect, and its increasing expression is associated with cerebrovascular diseases (51). Decreasing expression of MMP-9 inhibits aseptic inflammation and BBB destruction to a certain extent (52), and avoids neuronal damage and apoptosis (53). The results show that MMP-9 expression increased significantly, indicating that it has a key role during MCAO.

TIMPs are specific endogenous inhibitors of MMPs (54). Cysteine residues in the active site of TIMPs combine with the zinc-ion active centre of activated MMPs to inhibit MMP binding with substrates, preventing ECM degradation (55). Therefore, TIMPs are a potential target for the treatment of cerebral ischaemia-reperfusion (56).

MMPs and TIMPs are key factors that regulate the BBB integrity (57). Cerebrovascular occlusion results in secondary haemorrhage and brain oedema due to BBB destruction, plasma extravasation and leukocyte infiltration (58-60). It was reported $\mathrm{BBB}$ destruction in the present MCAO model increased the degree of neurological impairment and cerebral infarction (61). In the current study, the expression of MMP-9, TIMP-1 and COL IV changed remarkably after SJZD treatment. These studies suggest that TIMPs and MMPs may mediate the anti-anoikis mechanism of SJZD treatment via ECM stabilization in cerebral ischaemia-reperfusion.

The present study found that the permeability of the BBB in the hippocampus, as well as MMP-9 expression and apoptotic rate increased following MCAO, and COL IV expression significantly decreased. The increased expression of TIMP-1 after MCAO was consistent with previous reports $(62,63)$. SJZD treatment decreased BBB permeability, MMP-9 expression and apoptotic rate, and increased COL IV and TIMP-1 expression. The higher dose of SJZD was found to be as effective as nimodipine. These results suggest that this invigorating spleen therapy may protect neurons in cerebral ischaemia-reperfusion via stabilization of the ECM, particularly when used at a higher dose. These data are preliminary, and further studies are needed to confirm the presented results.

\section{Acknowledgements}

Not applicable.

\section{Funding}

This work was supported by grants from the National Natural Science Foundation of China (nos. 81373551,81473567 and 81603512), Key Science and Research Program of Hunan Department of Science and Technology (no. 60010408), Project of Hunan Provincial Education Department (no. 14B134) and China Postdoctoral Science Foundation (nos. 2017T100604 and 2016M600632).

\section{Availability of data and materials}

The datasets used and/or analyzed during the current study are available from the corresponding author on reasonable request.

\section{Authors' contributions}

LL and HYH designed the study.PY, YMT and WXD performed the experiments. WHL participated in the study design and data collection. PY and XC performed statistical analyses. PY wrote the manuscript, and LL revised the manuscript.

\section{Ethics approval and consent to participate}

The current study was approved by the Brains Hospital of Hunan Province Ethics Committee (grant no. Z2015003).

\section{Patient consent for publication}

Not applicable.

\section{Competing interests}

The authors declare that they have no competing interests.

\section{References}

1. Arnberg F, Grafström J, Lundberg J, Nikkhou-Aski S, Little P, Damberg P, Mitsios N, Mulder J, Lu L, Söderman M, et al: Imaging of a clinically relevant stroke model: Glucose hypermetabolism revisited. Stroke 46: 835-842, 2015.

2. Jickling GC, Liu D, Ander BP, Stamova B, Zhan X and Sharp FR: Targeting neutrophils in ischemic stroke: Translational insights from experimental studies. J Cereb Blood Flow Metab 35: 888-901, 2015.

3. Meschia JF, Bushnell C, Boden-Albala B, Braun LT, Bravata DM, Chaturvedi S, Creager MA, Eckel RH, Elkind MS, Fornage M, et al: Guidelines for the primary prevention of stroke: A statement for healthcare professionals from the American Heart Association/American Stroke Association. Stroke 45: 3754-3832, 2014

4. Lv Z, Liu C, Zhai M, Zhang Q, Li J, Zheng F and Peng M: LPS Pretreatment attenuates cerebral ischaemia/reperfusion injury by inhibiting inflammation and apoptosis. Cell Physiol Biochem 45: 2246-2256, 2018.

5. Carneiro BR, Pernambuco Filho PC, Mesquita AP, da Silva DS, Pinhal MA, Nader HB and Lopes CC: Acquisition of anoikis resistance up-regulates syndecan-4 expression in endothelial cells. PLoS One 9: e116001, 2014.

6. Hill JW, Poddar R, Thompson JF, Rosenberg GA and Yang Y: Intranuclear matrix metalloproteinases promote DNA damage and apoptosis induced by oxygen-glucose deprivation in neurons. Neuroscience 220: 277-290, 2012.

7. Sater AP, Rael LT, Tanner AH, Lieser MJ, Acuna DL, Mains CW and Bar-Or D: Cell death after traumatic brain injury: Detrimental role of anoikis in healing. Clin Chim Acta 482: 149-154, 2018.

8. Guo Z, Sun X, He Z, Jiang Y and Zhang X: Role of matrix metalloproteinase-9 in apoptosis of hippocampal neurons in rats during early brain injury after subarachnoid hemorrhage. Neurol Sci 31: 143-149, 2010.

9. Frisch SM, Schaller M and Cieply B: Mechanisms that link the oncogenic epithelial-mesenchymal transition to suppression of anoikis. J Cell Sci 126: 21-29, 2013.

10. Mazumder MK, Bhattacharya P and Borah A: Inhibition of matrix metalloproteinase- 2 and 9 by Piroxicam confer neuroprotection in cerebral ischemia: An in silico evaluation of the hypothesis. Med Hypotheses 83: 697-701, 2014.

11. Cheema ZF, Santillano DR, Wade SB, Newman JM and Miranda RC: The extracellular matrix, p53 and estrogen compete to regulate cell-surface Fas/Apo-1 suicide receptor expression in proliferating embryonic cerebral cortical precursors, and reciprocally, Fas-ligand modifies estrogen control of cell-cycle proteins. BMC Neurosci 5: 11, 2004.

12. Tian G, Wu C, Li J, Liang B, Zhang F, Fan X, Li Z, Wang Y, Li Z, Liu D, et al: Network pharmacology based investigation into the effect and mechanism of modified sijunzi decoction against the subtypes of chronic atrophic gastritis. Pharmacol Res 144: 158-166, 2019. 
13. Li J, Qian J, Jia JG, Jin X, Yu DJ, Xie B, Qian LY, Zhang LG and Guo CX: Effect of Sijunzi decoction on the proliferation of side population cells of human gastric cancer cell line. Zhongguo Zhong Xi Yi Jie He Za Zhi 34: 704-709, 2014 (In Chinese).

14. Hu KL, Li H, Liu WH, He Q, Zhong YL and Peng ZY: Effect of modified Si Junzitang on expression of ERK1/2, Akt, Bax in rats with cerebral ischemia/reperfusion injury. Chin J Exp Traditional Med Formulae 24: 152-158, 2008 (In Chinese)

15. Liu S, Zhang YB, Cao WG, Zhang D and Liu L: Comparision of the effects of three Chinese medicinal recipes to cerebral ischemia. Pharmacol Clin Chin Materia Medica 25: 6-7, 2009 (In Chinese)

16. Liu F and McCullough LD: Middle cerebral artery occlusion model in rodents: Methods and potential pitfalls. J Biomed Biotechnol 2011: 464701, 2011.

17. Ng YS, Stein J, Ning M and Black-Schaffer RM: Comparison of clinical characteristics and functional outcomes of ischemic stroke in different vascular territories. Stroke 38: 2309-2314, 2007.

18. Chinese Pharmacopoeia Commission: Pharmacopoeia of the People's Republic of China. Vol 1. 2015th edition. Chinese Medical Science and Technology Press, Beijing, pp8-240, 2015.

19. Longa EZ, Weinstein PR, Carlson $S$ and Cummins R: Reversible middle cerebral artery occlusion without craniectomy in rats. Stroke 20: 84-91, 1989.

20. Zhang Q, Zhou M, Wu X, Li Z, Liu B, Gao W, Yue J and Liu T: Promoting therapeutic angiogenesis of focal cerebral ischemia using thrombospondin-4 (TSP4) gene-modified bone marrow stromal cells (BMSCs) in a rat model. J Transl Med 17: 111, 2019.

21. Hao FL, Han XF, Wang XL, Zhao ZR, Guo AH, Lu XJ and Zhao XF: The neurovascular protective effect of alogliptin in murine MCAO model and brain endothelial cells. Biomed Pharmacother 109: 181-187, 2019.

22. Xu JH, Huang YM, Ling W, Li Y, Wang M, Chen XY, Sui Y and Zhao HL: Wen Dan Decoction for hemorrhagic stroke and ischemic stroke. Complement Ther Med 23: 298-308, 2015.

23. Hu HX, Lin RH, Zhu XQ, Li ZF and Chen LD: Anti-inflammatory effects of Gualou Guizhi decoction in transient focal cerebral ischemic brains [Corrected]. Mol Med Rep 12: 1321-1327, 2015.

24. Guo Q, Zhong M, Xu H, Mao X, Zhang Y and Lin N: A systems biology perspective on the molecular mechanisms underlying the therapeutic effects of buyang huanwu decoction on ischemic stroke. Rejuvenation Res 18: 313-325, 2015.

25. Zhang DS, Liu YL, Zhu DQ, Huang XJ and Luo CH: Point application with angong niuhuang sticker protects hippocampal and cortical neurons in rats with cerebral ischemia. Neural Regen Res 10: 286-291, 2015.

26. Zhang C, Wen Y, Fan XN, Tian G, Zhou XY, Deng SZ and Meng ZH: Therapeutic effects of different durations of acupuncture on rats with middle cerebral artery occlusion. Neural Regen Res 10: 159-164, 2015.

27. Zhong YL, Li H, Liu WH and Hu D: The effect of spleen-strengthening therapy on the mRNA and protein expression levels of INT $\beta 3$, ILK and FAK in Rats with cerebral ischemia/reperfusion injury. Lishizhen Med Mat Med Res 28: 513-516, 2017 (In Chinese)

28. Li H, Liu WH, Zhou XQ and He Q: Effects of spleen-strengthening therapy on MMP2 expression in brain tissue and blood brain barrier permeability in rats with cerebral ischemia/reperfusion injury. Hunan J TCM 29: 115-117, 2013.

29. Li H, Liu WH, Liao LY, Liu BY and Cai GX: Effect of tonifying spleen therapy on the degradation of laminin in rats with cerebra ischemia-reperfusion. Chin J Geriatr Heart Brain Vessel Dis 12 645-647, 2010 (In Chinese)

30. Adamczak JM, Schneider G, Nelles M, Que I, Suidgeest E, van der Weerd $\mathrm{L}$, Löwik $\mathrm{C}$ and Hoehn $\mathrm{M}$ : In vivo bioluminescence imaging of vascular remodeling after stroke. Front Cell Neurosci 8: 274, 2014

31. Reinhard J, Renner M, Wiemann S, Shakoor DA, Stute G, Dick HB, Faissner A and Joachim SC: Ischemic injury leads to extracellular matrix alterations in retina and optic nerve. Sci Rep 7: 43470, 2017.

32. Ravi S, Caves JM, Martinez AW, Xiao J, Wen J, Haller CA, Davis ME and Chaikof EL: Effect of bone marrow-derived extracellular matrix on cardiac function after ischemic injury. Biomaterials 33: 7736-7745, 2012.

33. Ma NK, Lim JK, Leong MF, Sandanaraj E, Ang BT, Tang C and Wan AC: Collaboration of 3D context and extracellular matrix in the development of glioma stemness in a 3D model. Biomaterials 78: 62-73, 2016
34. Klingberg F, Chow ML, Koehler A, Boo S, Buscemi L, Quinn TM, Costell M, Alman BA, Genot E and Hinz B: Prestress in the extracellular matrix sensitizes latent TGF- $\beta 1$ for activation. J Cell Biol 207: 283-297, 2014.

35. Faissner A and Reinhard J: The extracellular matrix compartment of neural stem and glial progenitor cells. Glia 63: 1330-1349, 2015.

36. Dzyubenko E, Gottschling C and Faissner A: Neuron-glia interactions in neural plasticity: Contributions of neural extracellular matrix and perineuronal nets. Neural Plast 2016: 5214961, 2016

37. Pandey AK, Bhattacharya P, Shukla SC, Paul S and Patnaik R: Resveratrol inhibits matrix metalloproteinases to attenuate neuronal damage in cerebral ischemia: A molecular docking study exploring possible neuroprotection. Neural Regen Res 10: 568-575, 2015

38. Neuwelt EA, Bauer B, Fahlke C, Fricker G, Iadecola C, Janigro D, Leybaert L, Molnár Z, O'Donnell ME, Povlishock JT, et al: Engaging neuroscience to advance translational research in brain barrier biology. Nat Rev Neurosci 12: 169-182, 2011.

39. Limmer S, Weiler A, Volkenhoff A, Babatz F and Klämbt C: The Drosophila blood-brain barrier: Development and function of a glial endothelium. Front Neurosci 8: 365, 2014

40. Ronaldson PT and Davis TP: Blood-brain barrier integrity and glial support: Mechanisms that can be targeted for novel therapeutic approaches in stroke. Curr Pharm Des 18: 3624-3644, 2012.

41. Wong AD, Ye M, Levy AF, Rothstein JD, Bergles DE and Searson PC: The blood-brain barrier: An engineering perspective. Front Neuroeng 6: 7, 2013

42. Hladky SB and Barrand MA: Fluid and ion transfer across the blood-brain and blood-cerebrospinal fluid barriers; a comparative account of mechanisms and roles. Fluids Barriers CNS 13 19, 2016.

43. Sazonova OV, Isenberg BC, Herrmann J, Lee KL, Purwada A, Valentine AD, Buczek-Thomas JA, Wong JY and Nugent MA: Extracellular matrix presentation modulates vascular smooth muscle cell mechanotransduction. Matrix Biol 41: 36-43, 2015.

44. Li S, Jin Z, Koirala S, Bu L, Xu L, Hynes RO, Walsh CA, Corfas $\mathrm{G}$ and Piao X: GPR56 regulates pial basement membrane integrity and cortical lamination. J Neurosci 28: 5817-5826, 2008.

45. Johnson KM, Milner R and Crocker SJ: Extracellular matrix composition determines astrocyte responses to mechanical and inflammatory stimuli. Neurosci Lett 600: 104-109, 2015.

46. Moore MC, Pandolfi V and McFetridge PS: Novel human-derived extracellular matrix induces in vitro and in vivo vascularization and inhibits fibrosis. Biomaterials 49: 37-46, 2015.

47. Zhang Z, Wang F, Wang BJ, Chu G, Cao Q, Sun BG and Dai QY: Inhibition of leptin-induced vascular extracellular matrix remodelling by adiponectin. J Mol Endocrinol 53: 145-154, 2014.

48. Xie Y, Mustafa A, Yerzhan A, Merzhakupova D, Yerlan P, N Orakov A, Wang X, Huang Y and Miao L: Nuclear matrix metalloproteinases: Functions resemble the evolution from the intracellular to the extracellular compartment. Cell Death Discov 3: 17036, 2017

49. Chopra K, Baveja A and Kuhad A: MMPs: A novel drug target for schizophrenia. Expert Opin Ther Targets 19: 77-85, 2015

50. Fu S, Gu Y, Jiang JQ, Chen X, Xu M, Chen X and Shen J: Calycosin-7-O- $\beta$-D-glucoside regulates nitric oxide/caveolin-1/matrix metalloproteinases pathway and protects blood-brain barrier integrity in experimental cerebral ischemia-reperfusion injury. J Ethnopharmacol 155: 692-701, 2014.

51. Wang JJ, Huan SK, Hsieh KH, Chou HC, Hsiao G, Jayakumar T and Sheu JR: Inhibitory effect of midazolam on MMP-9, MMP-1 and MMP-13 expression in PMA-stimulated human chondrocytes via recovery of NF-kB signaling. Arch Med Sci 9: 332-339, 2013.

52. He ZJ, Huang ZT, Chen XT and Zou ZJ: Effects of matrix metalloproteinase 9 inhibition on the blood brain barrier and inflammation in rats following cardiopulmonary resuscitation. Chin Med J (Engl) 122: 2346-2351, 2009.

53. Kwon M, Seo S, Chun H, Chung JM, Chung IK and Hur KC: Dual effect of nerve growth factor on cell death of PC12 cells Induced by serum deprivation. Mol Cells 13: 167-174, 2002.

54. Kim YS and Joh TH: Matrix metalloproteinases, new insights into the understanding of neurodegenerative disorders. Biomol Ther (Seoul) 20: 133-143, 2012. 
55. Visse R and Nagase $\mathrm{H}$ : Matrix metalloproteinases and tissue inhibitors of metalloproteinases: Structure, function, and biochemistry. Circ Res 92: 827-839, 2003.

56. Li JS, Liu K, Liu JX, Wang MH, Zhao YW and Liu ZG: Relationship between the changes in ischemia/reperfusion cerebro-microvessel basement membrane injury and gelatinase system in senile rat. Zhongguo Wei Zhong Bing Ji Jiu Yi Xue 20: 656-659, 2008 (In Chinese).

57. Michalski D, Hobohm C, Weise C, Pelz J, Heindl M, Kamprad M, Kacza J and Härtig W: Interrelations between blood-brain barrier permeability and matrix metalloproteinases are differently affected by tissue plasminogen activator and hyperoxia in a rat model of embolic stroke. Med Gas Res 2: 2, 2012

58. Liu XR, Luo M, Yan F, Zhang CC, Li SJ, Zhao HP, Ji XM and Luo YM: Ischemic postconditioning diminishes matrix metalloproteinase 9 expression and attenuates loss of the extracellular matrix proteins in rats following middle cerebral artery occlusion and reperfusion. CNS Neurosci Ther 18: 855-863, 2012.

59. Gu Y, Dee CM and Shen J: Interaction of free radicals, matrix metalloproteinases and caveolin-1 impacts blood-brain barrier permeability. Front Biosci (Schol Ed) 3: 1216-1231, 2011.
60. Zhu M, Xing D, Lu Z, Fan Y, Hou W, Dong H, Xiong L and Dong H: DDR 1 may play a key role in destruction of the blood-brain barrier after cerebral ischemia-reperfusion. Neurosci Res 96: 14-19, 2015

61. Tan F, Fu W, Cheng N, Meng DI and Gu Y: Ligustrazine reduces blood-brain barrier permeability in a rat model of focal cerebral ischemia and reperfusion. Exp Ther Med 9: 1757-1762, 2015.

62. Li DD, Song JN, Huang H, Guo XY, An JY, Zhang M, Li Y, Sun P, Pang HG, Zhao YL and Wang JF: The roles of MMP-9/TIMP-1 in cerebral edema following experimental acute cerebral infarction in rats. Neurosci Lett 550: 168-172, 2013.

63. Lenglet S, Montecucco F, Mach F, Schaller K, Gasche Y and Copin JC: Analysis of the expression of nine secreted matrix metalloproteinases and their endogenous inhibitors in the brain of mice subjected to ischaemic stroke. Thromb Haemost 112: 363-378, 2014.

This work is licensed under a Creative Commons Attribution-NonCommercial-NoDerivatives 4.0 International (CC BY-NC-ND 4.0) License. 